\title{
2D ultrasonic elastography with lateral displacement estimation using statistics
}

\author{
Zhihong Zhang ${ }^{\mathrm{a}}$, Haolin Liu ${ }^{\mathrm{b},{ }^{*}}$ and Yangjie Cheng ${ }^{\mathrm{c}}$ \\ ${ }^{a}$ Department of Computer Science and Technology, Changsha College, Changsha 410003, China \\ ${ }^{\mathrm{b}}$ School of Computer Science, Sichuan University, Chengdu, Sichuan 610065, China \\ ${ }^{c}$ Detection \& Guidance Solution, GE Healthcare, Chengdu, Sichuan 611731, China
}

\begin{abstract}
Ultrasound elastography is the method of obtaining relative stiffness information of biological tissue, which plays an important role in early diagnosis. Generally, a gradient-based strain imaging algorithm assumes that motion only occurs in an axial direction. However, because tissue has different relative stiffness, the scatter presents lateral motion under high freehand compression. Therefore, errors occur in estimating the cross-correlation phase in the calculation window. A 2D elastography algorithm with lateral displacement estimation using statistics was proposed to reduce errors. The new method was investigated through simulation, and the experiment confirmed that errors introduced by lateral tissue movement have been greatly reduced with no sacrifice of real-time ultrasonic imaging quality.
\end{abstract}

Keywords: 2D elastography, lateral motion, cross-correlation, statistics

\section{Introduction}

Elastography, the imaging modality of the elastic properties of biological tissue using ultrasound, was firstly developed by Ophir, et al. in 1991 [1]. Generally, light external mechanical pressure is applied on soft tissue, and an axial strain calculation compares the signal before the application of force, or the pre-compression signal, with the post-compression signal. An elastogram is calculated as the gradient of the axial displacements and displayed as a gray scale image where the dark areas correspond to hard tissue and the light areas indicate soft tissue. With ultrasonic elastography, elastic properties are measured quantitatively, which offers more information than qualitative analysis of palpation.

Two main concepts exist for gradient-based strain imaging: the correlation technique in time domain [2-5] and the phase domain [6-8]. Phase estimation has low calculation costs in obtaining displacement. One commonly used algorithm is phase zero estimation developed by Ophir, et al., but it only considers axial displacements. Because tissue has different relative stiffness, free-hand compression usually causes scattering of the lateral displacement, which causes decorrelation errors in the axial calculation in the phase zero algorithm (PZA), especially when high compression is applied.

\footnotetext{
${ }^{*}$ Corresponding author: Haolin Liu, School of Computer Science, Sichuan University, Chengdu, Sichuan, 610065, China. Tel.:13408565115; Email: liuhaolin8828@163.com.
} 
In order to reduce decorrelation errors in the PZA, the modified phase zero algorithm (MPZA) [9] was introduced by Jiang, et al. It makes use of information from multiple scan lines and expands a 1D window to a $2 \mathrm{D}$ window to enhance the correlation between windows and reduce side-slipping of the 1D axial signal. The phase zero algorithm depends on an initial value, thus follow-up calculation will be greatly affected by the initial value. Consequently, if the initial value is not accurate, it will lead to errors in the axial displacement estimation.

One good method to reduce errors in phase zero estimation is to revise the iterative initial value, lowering the accumulated errors brought by iteration. This paper introduces a new method that revises the iterative initial value by using lateral statistics in the calculating window. Through simulation and in vivo data experiments, it showed that errors can be efficiently reduced by the proposed method.

\section{Methods}

\subsection{Modified phase zero algorithm (MPZA)}

Let $x_{1}$ and $x_{2}$ represent the pre-compression and post-compression echo signals, respectively, and $x_{2}$ can be considered a time-shifted version of $x_{1}$. Assume that in a given time range around the signal is rigidly shifted. Thus $x_{2}$ can be shown by:

$$
x_{2}(t)=x_{1}(t+\tau)
$$

where $\tau$ is the time shift. Consequently, the cross-correlation function is a time-shifted version of the autocorrelation function:

$$
\left\langle x_{1}, x_{2}\right\rangle(t)=\left\langle x_{1}, x_{1}\right\rangle(t+\tau)
$$

Discrete time delay estimation gives:

$$
\begin{aligned}
& \tau_{0,0}=0, \tau_{k, 0}=\tau_{k-1, N} \\
& \tau k, l_{x, y}=\tau_{k, l-1}-\frac{1}{\omega_{0}} \arg \left(e^{j \omega_{0} \tau_{k, l-1}} \int_{\tau_{k, l-1-1} T_{a} / 2}^{\tau} x_{1 b}(t) x_{2 b}^{*}\left(t-\tau_{k, l-1}\right) d t\right)
\end{aligned}
$$

where $\tau_{k, l}$ represents the displacements in the kth window after iterating for $l$ times, TW represents the window size used for cross-correlation, and operator $*$ denotes the conjugate of the complex.

Original phase zero estimation makes the assumption that tissue displacement only takes place in the axial direction. However, scatters normally have lateral movements when freehand compression is applied. This leads to low signal correlation in the window across a scan line, and as a result, increases errors in the cross-correlation phase calculation. This problem can be partially solved by the modified phase zero algorithm, which gives: 


$$
\begin{aligned}
& \tau_{x, 0}=0 \\
& \tau_{x, y}=\tau_{x, y-1}-\frac{1}{\omega_{0}} \arg \left(e^{j \omega_{0} \tau_{x, y-1}} \int_{-T_{1} / 2}^{T_{1} / 2} \int_{x, y-1-1 / 2}^{\tau_{x, y-1} \tau_{a} T_{a} / 2} x_{1 b}\left(x, t+\frac{\tau_{x, y}}{2}\right) x_{2 b}^{*}\left(x, t-\frac{\tau_{x, y-1}}{2}\right) d t d x\right)
\end{aligned}
$$

where $\mathrm{x}$ and $\mathrm{y}$ refer to lateral direction and axial direction, respectively, $\tau_{x, y}$ is the estimated displacement in $\mathrm{x} t h$ point on $\mathrm{yth}$ scan line, and Ty and Tx refer to the length of the axial temporal window and lateral temporal window, respectively.

\section{2. $2 D$ modified phase zero algorithm with initial value revising (2D PZA)}

When tissue is compressed, the axial displacements between nearby scan lines are highly correlated. Assume that axial displacements change very little among nearby scan lines. Then displacement estimation results from the statistics windows are counted and the erroneous displacement result is excluded. Then, average displacements of the result will be applied as the initial value for the next window's phase zero estimation. These principles can be better understood by referring to Figure 1 .

Phase zero estimation usually gives low displacement results (absolute value) when calculated incorrectly. This can cause large errors in averaging the displacements from the statistics window. In order to achieve a stable initial value, the minimum (absolute) value is discarded when performing the average calculation, which is used in the next group of windows. After adding the initial value revision, 2D PZA can be described as:

$$
\begin{aligned}
& \tau_{x, 0}=0 \\
& \tau_{x, y-1}=\operatorname{avg}\left(\tau_{x-T_{1} / 2, y-1} \ldots \tau_{x+T_{1} / 2, y-1}-\min \left(\operatorname{abs}\left(\left[\tau_{x-T_{1} / 2, y-1} \ldots \tau_{x+\frac{T}{1} / 2, y-1}\right]\right)\right)\right. \\
& \tau_{x, y}=\tau_{x, y-1}-\frac{1}{\omega_{0}} \arg \left(e^{j \omega_{0} \tau_{x-1, y-1}} \int_{-T_{T} / 2}^{T_{1} / 2} \tau_{x-1, y-1-1 / 2}^{\tau_{x-1, y-1+2} T_{a} / 2} x_{1 b}(x, t) x_{2 b}^{*}\left(x, t-\tau_{x, y-1}\right) d t d x\right) \\
& \begin{array}{llll}
\multicolumn{9}{c}{\text { Lateral } \mathrm{x}} \\
\text { Statistics Window }
\end{array}
\end{aligned}
$$

Fig. 1. Lateral revising principles using statistics. 
where min refers to minimum value. Different displacements among each scan line increases with depth. If the large statistics window is used in distant fields, the average result will deviate from the real displacement. Therefore, the statistics window size must be self-adaptive based on depth. For example, a large window size should be used in a near field and a small window size should be used in a distant field. This can ensure the axial resolution of strain estimation L using Eq. (5):

$$
L=\frac{(M-m+1) L_{0}}{M}
$$

where $\mathrm{M}$ refers to the total number of axial windows, $\mathrm{m}(\mathrm{m}=1 . . \mathrm{M})$ refers to $m$ th axial window, $\mathrm{L} 0$ refers to the initial statistics window size, and $\mathrm{L}$ refers to the statistics window size.

The flow chart of the 2D modified phase zero algorithm with a revised initial value using the lateral statistics window is shown in Figure 2. As:

1. Taking the given parameters of the $2 \mathrm{D}$ window (size, overlap), divide the IQ data into $\mathrm{M} \times \mathrm{N}$ parts $(m=1 . . M)$. Set the initial iteration value for the statistics window on the first line to be 0 .

2. Calculate and record the displacement of the $m$ th axial estimation window of all scan lines using 2D phase zero estimation.

3. Slide the statistics window, calculate the displacements of the $m$ th axial window, exclude the erroneous displacement and average the rest of the displacements in the statistics window.

4. Use the results from the statistics window as the initial value of $m+1$ th axial window in all scan lines.

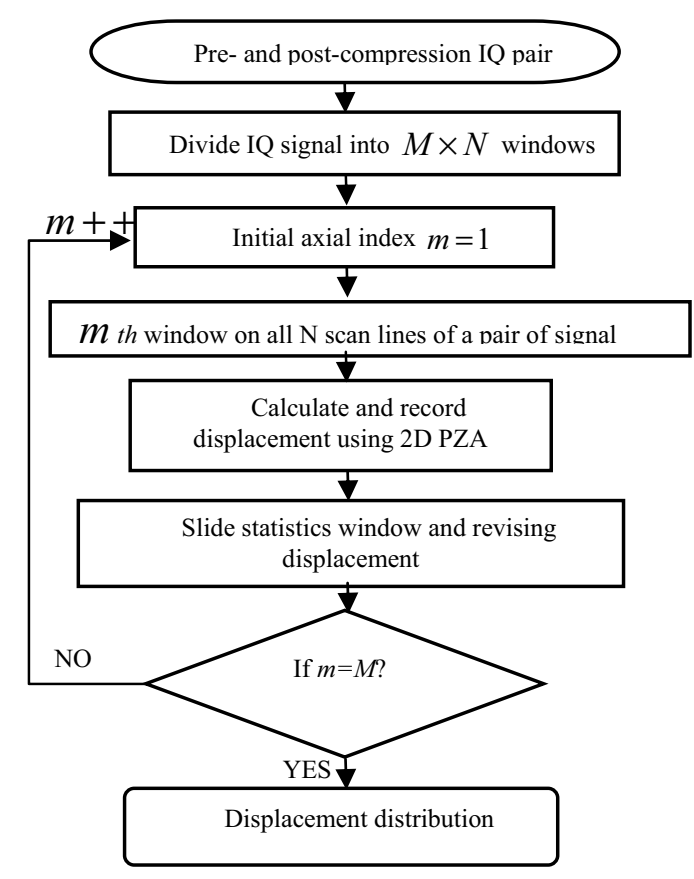

Fig. 2. Flow chart of 2D PZA with revised initial value.

5. Repeat numbers 2-4 until all the axial windows have been accounted for. 


\section{Experiment}

\subsection{Experiment platform}

In the simulation model experiment, a model introduced by Xunchang Chen [10] is shown in Figure 3 and the lateral and axial displacements were added. Multiple tissue models were designed with different compression ratios $P$. The signal amplitude was normalization and the PZA, MPZA, and 2D PZA were implemented on all the model signals to estimate tissue displacements. A SG-I DD digital differential filter [11-12] was used to acquire the displacements to calculate the strains. The IQ signal was sampled to $512 \times 300$; the axial window for the PZA was 25 with $80 \%$ overlap. Linear interpolation was taken and the number of iterations was 1 . The lateral statistics window was 4 in the 2D PZA. The range of the self-adaptive lateral window size was 11 to 5, which decreases when the depth deepens. The size of the differential filter was 7 .

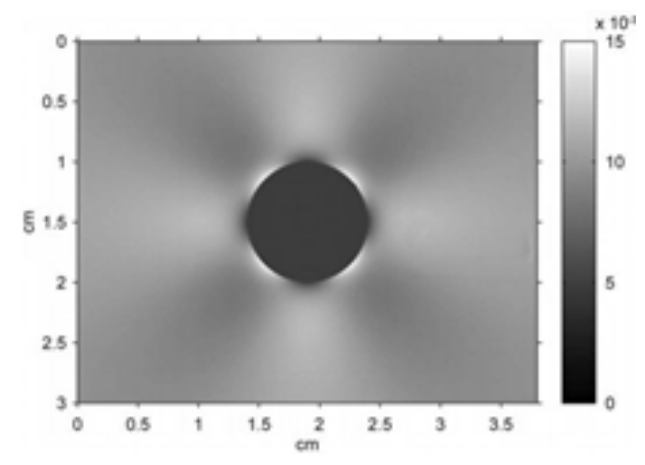

Fig. 3. Simulation model.

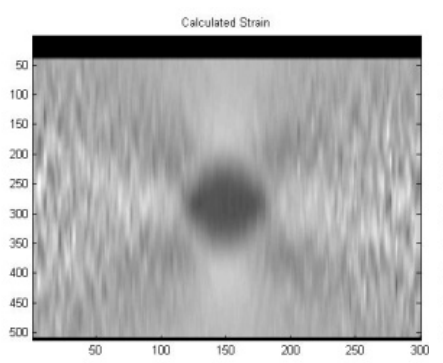

(a) PZA

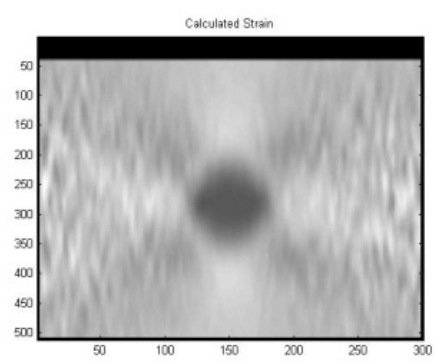

(b) MPZA

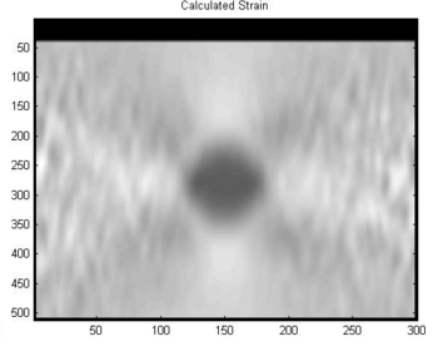

(c) 2D PZA

Fig. 4. Strain images of different algorithms when compression ratio is $1 \%$. 


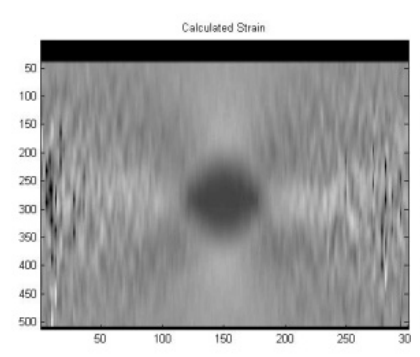

(a) PZA

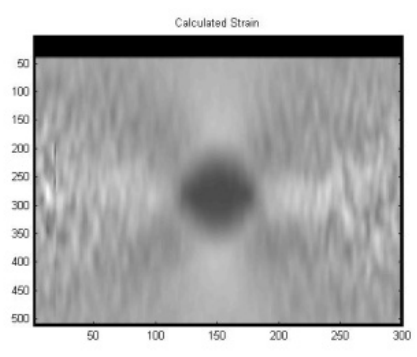

(b) MPZA

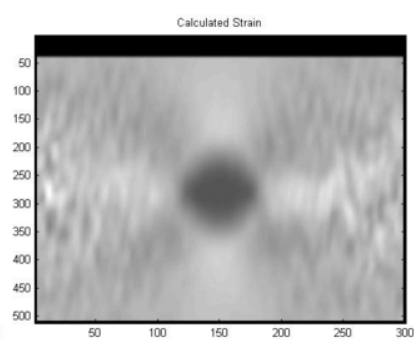

(c) 2D PZA

Fig. 5. Strain images of different algorithms when compression ratio is $2 \%$.

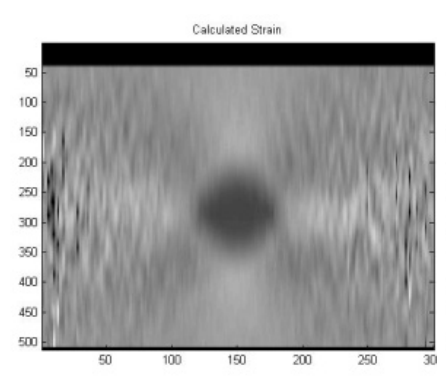

(a) PZA

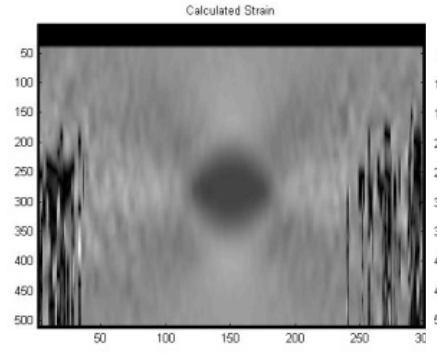

(b) MPZA

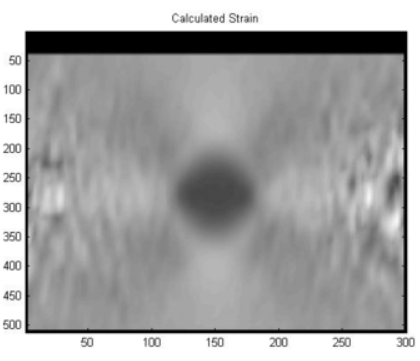

(c) 2D PZA

Fig. 6. Strain images of different algorithms when compression ratio is $3 \%$.

\subsection{Results}

Figures 4-7 show the strain images estimated by the PZA, MPZA, and 2D PZA with the revised initial value under different compression ratios.

The results obtained with the different compression ratios are discussed: When the compression ratio was $1 \%$ and $2 \%$, the targets could be detected clearly and errors in averaging displacements were low; when the compression ratio was $3 \%$, a small number of dark vertical stripes occurred locally in

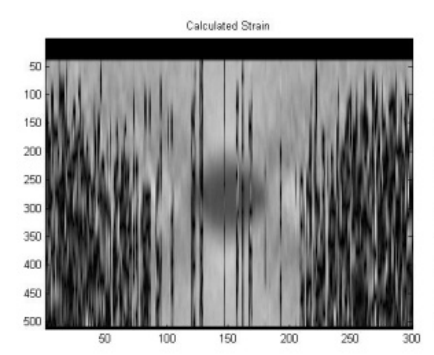

(a) PZA

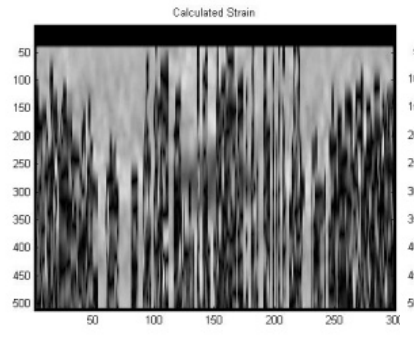

(b) MPZA

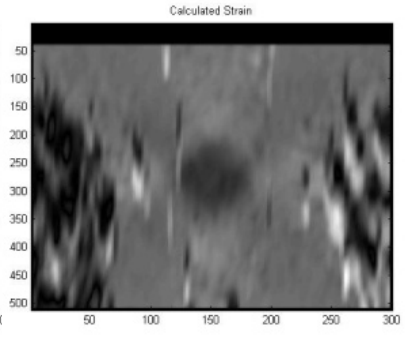

(c) 2D PZA

Fig. 7. Strain images of different algorithms when compression ratio is $4 \%$. 


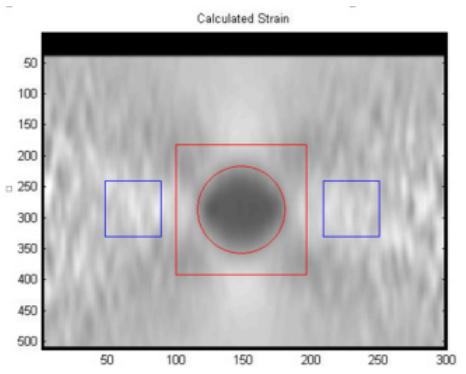

Fig. 8. Region of interest of strain image.

the areas of the results for the original PZA and the MPZA, interfering with the tissues on both sides. However, for the 2D PZA, the displacement was accurately calculated and such an error did not occur. When the compression ratio was $4 \%$, the dark vertical stripes of the strain images for the PZA and the MPZA were denser compared to the $3 \%$ compression ratio. Due to miscalculations using the PZA and the MPZA, the generated dark vertical stripes occurred in the location of the target lesion, seriously disrupting its detection. In the strain image obtained on the basis of the 2D PZA with the revised iterative initial value, errors were distributed in a small area and only concentrated on both sides, having no effect on the detection of the target.

In Figures 4-7, under high compression ratios (3\%-4\%), the traditional PZA and MPZA algorithms had large errors in the phase calculations, resulting in bad image qualities. In contrast, by amending the initial value, the 2D PZA with the revised initial value using lateral statistics could effectively reduce the errors. This method can be used to calculate tissue strain accurately and locate pathological tissue.

In order to quantitatively evaluate the image quality, the signal-to-noise of elastography (SNRe) and contrast-to-noise of elastography $(\mathrm{CNRe})$ of the strain image in Figure 8 were calculated. SNRe is defined as:

$$
S N R_{e}=\frac{\mu_{s}}{\sigma_{s}}
$$

where $\mu_{s}$ and $\sigma_{s}$ denote the mean and standard deviation of homogenous areas in the strain image.

CNRe is defined as:

$$
C N R_{e}=\frac{2\left(\mu_{s b}-\mu_{s t}\right)^{2}}{\sigma_{s b}{ }^{2}+\sigma_{s t}{ }^{2}}
$$

where $\mu_{s t}$ and $\mu_{s b}$ represent the mean of the target lesion and background tissue, $\mu_{s t}$ and $\mu_{s b}$ represent the variances of the target lesion and background tissue, respectively.

In Figure 9(a), when the compression ratio was between $1 \%$ and $4.5 \%$, the MPZA achieved higher SNRe than the PZA, which means that the MPZA had a smoother image than the PZA. This is because the MPZA involved more information by expanding the lateral width of the calculating window. The 


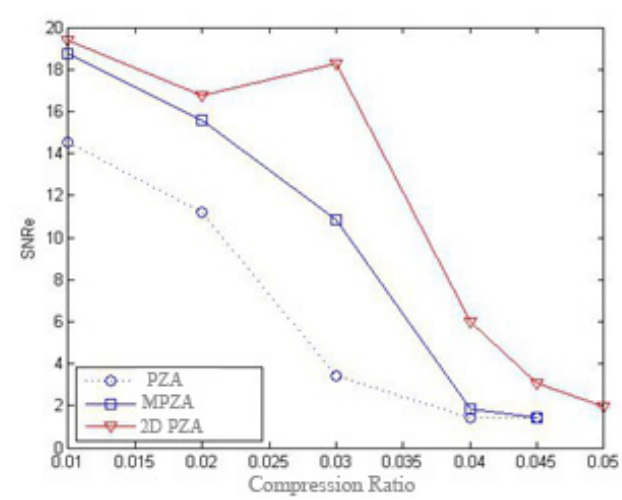

(a) SNRe

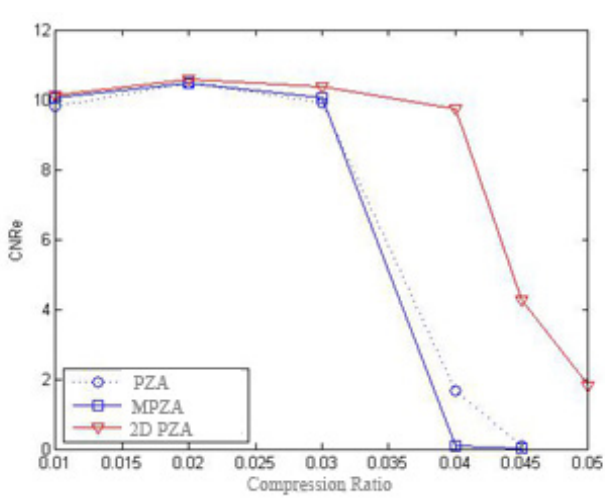

(b) CNRe

Fig. 9. Evaluation index of images under various compression ratios.

MPZA also had lower errors than the PZA. As for a revised initial value algorithm, it achieved higher SNRe than the other two algorithms.

With the 2D PZA, it was hard to judge whether the rigid deformation model failed and the initial value revision when rigidity did not fail was considered to produce an adverse effect. Therefore, only when the compression ratio was greater than $2 \%$ did the 2D PZA gradually come into play. Meanwhile, the SNRe was still higher when the compression ratio was above $3 \%$, which meant the $2 \mathrm{D}$ PZA with the revised initial value was more accurate than previous methods.

As can be seen in Figure 9(b), the three algorithms showed similar CNRe when the compression ratio was among $1 \%$ to $3 \%$. Notably, the $2 \mathrm{D}$ PZA with the revised initial value had a higher CNRe than the other two algorithms under all the compression ratios. It showed that the revised method had the ability to detect objects with low compression ratios as well as maintain high robustness with high compression ratios.

\section{Discussion}

The accurate estimation of displacement determines the success and precision of elastography. Quasi-static compression is the most commonly used technique in elastic imaging, which mainly utilizes phase-zero algorithms (PZA) to estimate displacements. The PZA estimates displacement by calculating the axial correlation of scan lines in two frames. One frame shows before quasi-static compression and the other is after the compression. However, the PZA assumes the tissue will respond with rigid deformation, and only move along the axial direction. Because this cannot be guaranteed in clinical settings, especially when freehand compression is applied, the PZA will have calculation errors, or even failure. A new method based on the MPZA was proposed, which revised the initial value of the iteration by using lateral statistics. Through a simulation model, it was proved that this method can achieve more accurate imaging and increase the robustness of displacement estimations. 


\section{References}

[1] J. Ophir, I. Céspedes, H. Ponnekanti, Y. Yazdi and X. Li, Elastography: a quantitative method for imaging the elasticity of biological tissue, Ultrasonic Imaging 13 (1991), 111-134.

[2] J. Ophir, I. Céspedes, B. Garra, H. Ponnekanti, Y. Huang and N. Maklad, Elastography: Ultrasonic imaging of tissue strain and elastic modulus in vivo, European Journal of Ultrasound 3 (1996), 49-70.

[3] J. Ophir, S.K. Alam, B. Garra, F. Kallel et al., Elastography: Ultrasonic estimation and imaging of the elastic properties of tissues, Journal of Engineering in Medicine 213 (1999), 203-233.

[4] J. Ophir, S.K. Alam, S. Brian, B.S. Garra, F. Kallel et al., Elastography: Imaging the elastic properties of soft tissues with ultrasound, Journal of Medical Ultrasound 29 (2002), 155-171.

[5] Joel E. Lindop, Graham M. Treece, Andrew H. Gee and Rihcar W. Prager, Phase-based ultrsounic deformation estimation, IEEE Trans. Ultrson., Ferroelect., Freq. Contr. 55 (2008), 94-110.

[6] A. Pesavento, C. Perrey, M. Krueger, et al., A time efficient and accurate strain estimation concept for ultrasonic elastography using iterative phase zero estimation, IEEE Trans Ultrason Ferroelec Freq Control 46 (1999), 1057-1067.

[7] J.E. Lindop, G.E. Treece, A. Gee, et al., Phase-based ultrasonic deformation estimation, IEEE Trans Ultrason Ferroelec Freq Control 55 (2008), 94-111.

[8] A. Ramalli, O. Basset, C. Cachard, et al., Frequency-domain-based strain estimation and high-frame-rate imaging for quasi-static elastography, IEEE Trans Ultrason Ferroelec Freq Control 59 (2012), 817-824.

[9] Z.Q. Jiang, P. Liu and D.C. Liu, Modified phase zero for ultrasound freehand strain imaging, In Proceedings of the 3rd iCBBE 2009, 68-73.

[10] Xunchang Chen, Stanislav Y. Emelianov and Matthew O'Donnell, Lateral speckle tracking using synthetic lateral phase, IEEE Trans Ultrason Ferroelec Freq Control, 51 (2004), 540-550.

[11] Peter N. T. Wells and Hai-Dong Liang, Medical ultrasound: imaging of soft tissue strain and elasticity, Journal of the Royal Society Interface 8 (2011), 1521-1549.

[12] Jianwen Luo, Jing Bai, Ping He, et al. Axial strain calculation using a low-pass digital differentiator in ultrasound elastography, IEEE Trans Ultrason Ferroelec Freq Control 51 (2004), 1119-1127. 